\title{
DESIGN STUDY OF THE HEAT SWITCH BASE PLATE WITH SINGLE AND MULTI-MATERIAL TOPOLOGY OPTIMIZATION
}

\author{
František Löffelmann ${ }^{1}$, Jan Šplíchal ${ }^{1}$ \\ ${ }^{1}$ Institute of Aerospace Engineering \\ Brno University of Technology \\ Technická 2, 61669 Brno, Czech Republic \\ Frantisek.Loffelmann@vutbr.cz \\ Jan.Splichal@vutbr.cz
}

Keywords: Stiffness, structural optimization, multiple materials, layer optimization, 3D printing, additive manufacturing.

\begin{abstract}
A miniaturized heat switch is a device under the development dedicated to control automatic cooling of a space instrument box. The design study presented in this paper focuses on a stiffness issue of the switch base plate which should assure good thermal contact with the instrument box. Due to inner deformations of the switch, base plate deforms which might decrease contact area needed to the heat transfer. Thus several design concepts of the copper base plate were investigated and multimaterial additive manufacturing was reviewed. One approach was to decrease deformations of existing geometry by multi-material design by local exchange of copper with steel, first by engineering intuition, then by the stiffness based topology optimization, and finally with the layer-wise topology optimization better respecting manufacturing possibilities. Another approach was to change the geometry and use the topology optimization constrained with a flatness of the base plate but with single material. Finally, deformation changes of all the variants were compared.
\end{abstract}

\section{INTRODUCTION}

Application of new technologies is expected to help with fulfilling high demands in space applications. One of such projects is the miniaturized heat switch which connects or disconnects an external heat exchanger with a box of thermally insulated instruments. The switch works on a purely mechanical principle where a paraffin filler changes its phase and volume which controls thermal connection between the hot and the cold plates as shown in Figure 1. The switch has to work in extreme space and Martian conditions as described by Mašek et al. [1] who developed the thermo-vacuum test chamber for the switch testing.

Major concern is that the switch must transfer heat from the hot plate to the cold plate when it is in the ON position. Another challenge is the contact quality between the instrument box and the switch. Paraffin pressure driving the actuator causes in the ON position swelling of the switch base plate so that contact surface might be significantly reduced. Our contribution investigates potential ways of stiffening base plate in order to increase contact area and thus heat transfer through the switch. We focused on two basic variants: first, reinforce the actual copper geometry with steel and second, modify overall geometry to use copper more efficiently (without second material). 


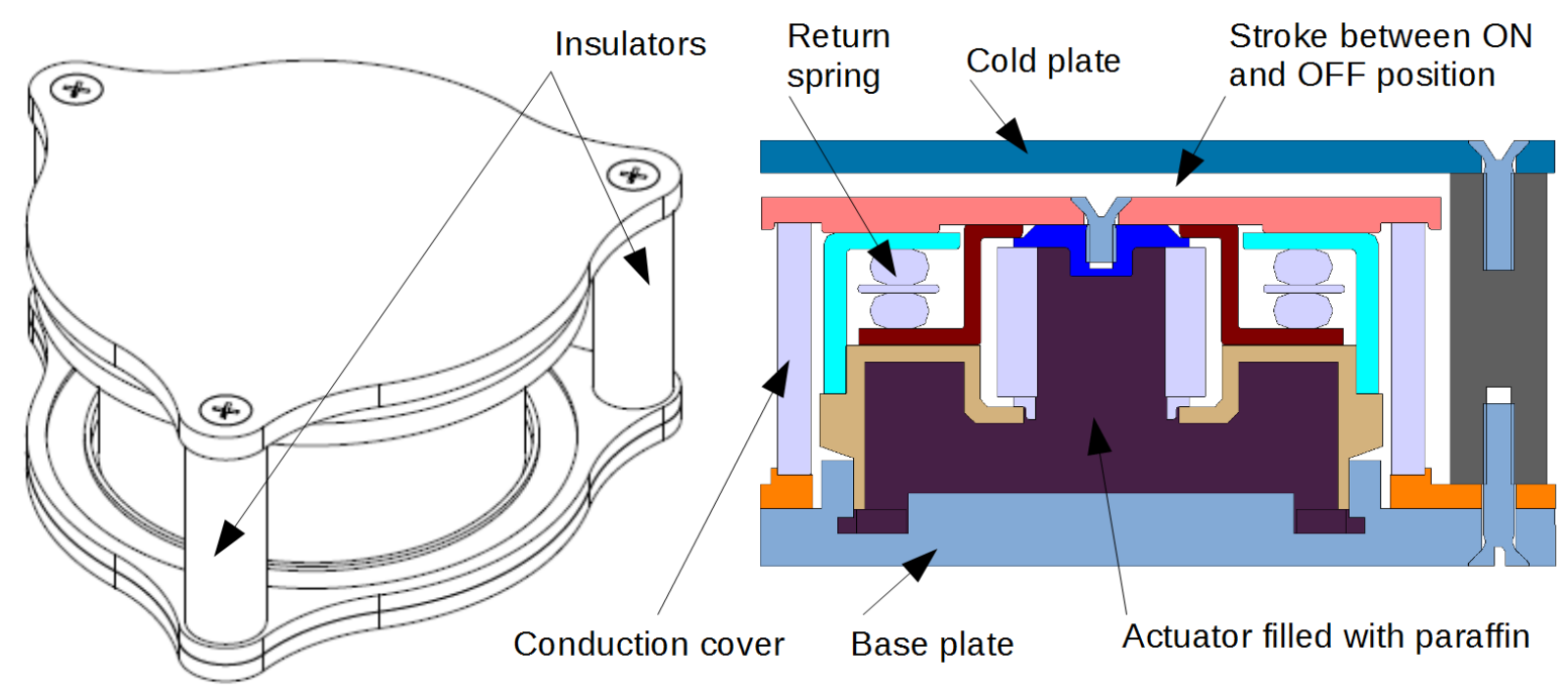

Figure 1: Miniaturized heat switch schema [2].

\section{SINGLE AND MULTI-MATERIAL ADDITIVE MANUFACTURING}

"Additive manufacturing (AM) is a process of joining materials to make objects from 3D model data, usually layer upon layer, as opposed to subtractive manufacturing methodologies," according to ASTM [3]. AM was recognized as a technology giving new opportunities and spreading into many branches, thanks to simple 3D printers for rapid prototyping up to advanced machines for high demand applications such as medical implants and aerospace parts [4].

Recent thorough review on AM was made by Ngo et al. [5]. Review of metal AM by Frazier [6] distinguishes three categories of AM: Powder bed systems; most common method in this category is selective laser melting (SLM) which uses laser as an energy source to melt powder in its layer which is repeatedly raked over the manufactured part. Second category is powder feed systems where powder is supplied through a nozzle directly to the part surface which facilitates larger components to build. Third category, wire feed systems have supply of a material in the form of wire to the heated spot which is also more productive but with lower accuracy.

AM enables combining multiple materials or produce special structures. Interesting category is functionally graded materials (FGM) [7] where one "material" gradually changes to another. This was initially used for material close to the surface with high thermal gradients. FGM are of three categories: chemical composition gradient FGM, porosity gradient FGM, and microstructure gradient FGM.

Bonding of several combinations of metallic materials was reported by Obielodan et al. [8] by use of ultrasonic consolidation of thin metallic plates, which forms solid object with temperatures less than half of the materials melting point. SLM with material change from layer to layer is possible with increased labor but using multiple materials in one layer needs special machines. Chivel [9] suggested a machine for SLS (selective laser sintering) or SLM which sinters/melts one material in the layer, then unused powder is removed from this layer by vacuum cleaner with brushes, and new powder is inserted to the same layer and sintered/melted. This process can be sequentially repeated for different materials before moving to the next layer. Using a powder size specific for each material facilitates separation and powder reuse. Recently, Wei et al. [10] manufactured samples combining 316L stainless steel, In718 nickel alloy and Cu10Sn copper alloy in one layer by use of modified SLM. First material was selectively melted in the layer of its powder. Powder from places for the next material was then removed by the micro-vacuum system and new material was locally dispensed there and melted by the laser beam. Studies showed that 3D metal multi-material AM is possible but not yet ready for industrial use. 


\section{TOPOLOGY OPTIMIZATION METHODS}

In this section topology optimization methods, which were used in our work, are briefly described.

\subsection{Single material}

Topology optimization with single material (usually minimizing compliance with volume constraint or minimizing mass with stress or displacement constraints) is already available in many commercial software. Most common is density method approach introduced by Bensøe [11]. Users guide [12] of MSC.Nastran, which we used for this optimization, does not deeply describe topology optimization theory but we can refer to the literature, e.g. Bensøe and Sigmund [13]. Commonly used SIMP (Solid Isotropic Material with Penalization) method transforms the discrete problem to the continuous with a pseudo-density between 0 and 1 (void and solid) as the design variable for each element which enables use of mathematical optimization methods such as MMA (Method of Moving Asymptotes). The pseudo-density is penalized with exponential coefficient which helps to converge close to discrete material values 0 or 1 .

\subsection{Multi-material}

Topology optimization of two material phases and void phase was reported already by Sigmund and Torquato [14] several years ago to solve extreme thermos-elastic properties. However, combining multiple materials is not still common due to manufacturing difficulties, and software tools for those tasks are also not widely available.

In our study we used simpler approach based on the BESO (Bi-directional Evolutionary Structural Optimization) method, which was recently comprehensively reviewed by Xia et al. [15]. We used implementation by the author [16] and the python code which is freely available. The method is based on the optimality criteria. Sensitivity number, which is taken as a measure of the element efficiency, was defined as the element strain energy density

$$
\alpha_{e}=\frac{1}{2} \boldsymbol{u}_{e} \boldsymbol{K}_{e} \boldsymbol{u}_{e}
$$

where $\mathbf{u}_{\mathrm{e}}$ is the element displacement vector and $\mathbf{K}_{\mathrm{e}}$ is the element stiffness matrix, which depends on the variable element material (weaker material 0 or stronger material 1 ). In each iteration prescribed volume $v_{01}$ of the material 0 is switched to the material 1 and in the opposite way $v_{10}$ prescribes volume to be switched from the material 1 to the material 0 . If $v_{01}>v_{10}$ than volume of the material 1 sequentially grows and the material 0 sequentially disappears. Selection of elements is evaluated according to their sensitivity number $\alpha_{\mathrm{e}}$. Elements with lowest $\alpha_{\mathrm{e}}$ candidate to switch down (from the stronger material 1 to the weaker material 0 ) because their stiffness contribution is lowest (low strain energy density caused by the loads). And in the opposite way, elements with highest $\alpha_{\mathrm{e}}$ candidate to switch up (from the weaker material 0 to the stronger material 1 ) because their stiffness contribution is highest. Since compliance is 2times strain energy, the model is kept on minimal compliance (maximal stiffness) while ratio of design materials is sequentially changing, i.e. we obtain minimal compliance results for the sequence of volume constraints. In the current study we monitored the model evolution and used small values of $v_{01}$ and $v_{10}$ without focus on the convergence in detail. Convergence criteria and more general explanation of the method were presented in the original paper [16].

\subsection{Multi-material - Layer-wise}

Layer-wise optimization is a modification of the previous approach. Elements are grouped, in our case into layers and switching was done for whole layers. In each iteration switching was limited only to switch material in one layer from the weaker material 0 to the material 1 according to sensitivity 
numbers calculated as the average from layer elements. Switching in the opposite direction was not allowed in this case.

\section{BASE PLATE OPTIMIZATION}

\subsection{Multi-material concepts}

\subsubsection{Intuitive approach}

Model of the base plate is in Figure 2 left. The original material was copper ( $E=110 \mathrm{GPa}, v=0.3$ ). Steel $(E=210 \mathrm{GPa}, \mathrm{v}=0.266$ ) was used as a reinforcement material arranged in four variants (Figure 2 right and Figure 3); reinforcements were chosen not to block thermal flow in vertical direction. FE model (Figure 2) was constrained in the locations of screws. The base plate was loaded with a pressure 11.937 MPa caused by the paraffin infill expansion (blue loaded surfaces are better visible in Figure 4).
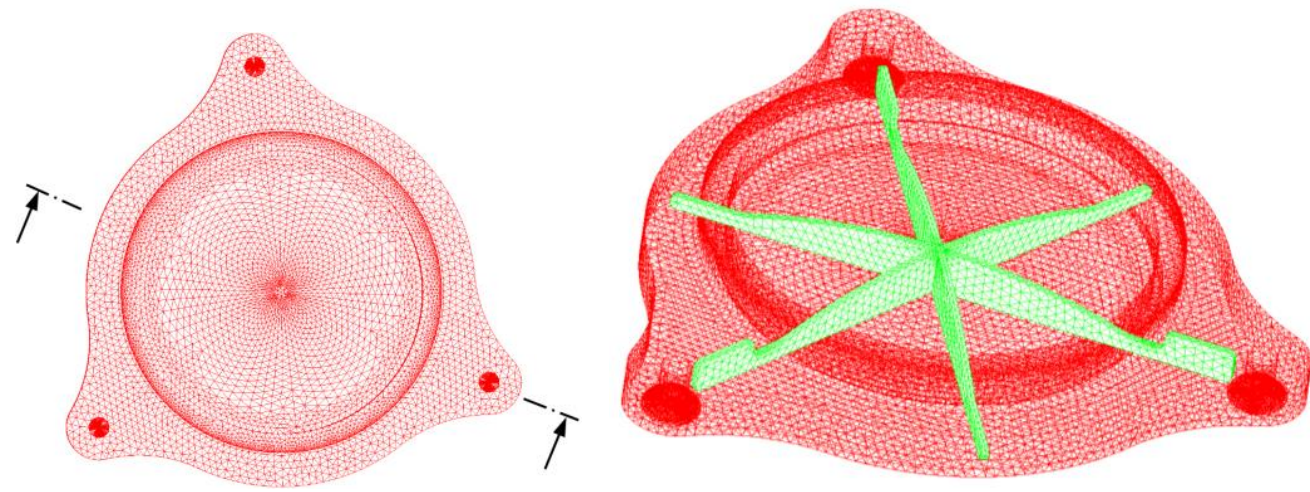

Figure 2: Basic model of the base plate (left), base plate with the inner stiffener (right).
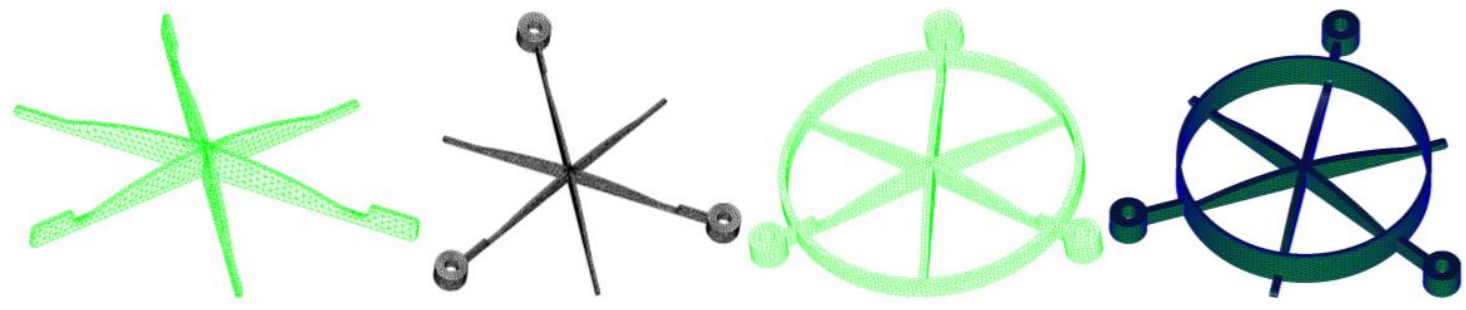

Figure 3: Stiffener variants (Model 01, Model 02, Model 03 and Model 04).

\subsubsection{Topology optimization}

Multi-material optimization aimed to minimize compliance with specific steel content in each iteration. Design domain covered whole base plate with material variables (copper as the material 0 and steel as the material 1) for each element. Model was prepared in PrePoMax, pre- and post-processor for CalculiX FE solver. One third of the base plate shown in Figure 4 was modelled with same material properties and pressure load as in the intuitive approach. Centre node deflection was measured. Five combinations of the mesh and optimization settings were used as noted in Table 1 (rows A1-B2). E.g. variant $A 2$ was set to growing of the steel reinforcement from initial copper model by $v_{01}=2 \%$ and $v_{10}$ $=1 \%$ which means that in each iteration 2 volume percent were switched from copper to steel and $1 \%$ was switched in the opposite way from steel to copper. 


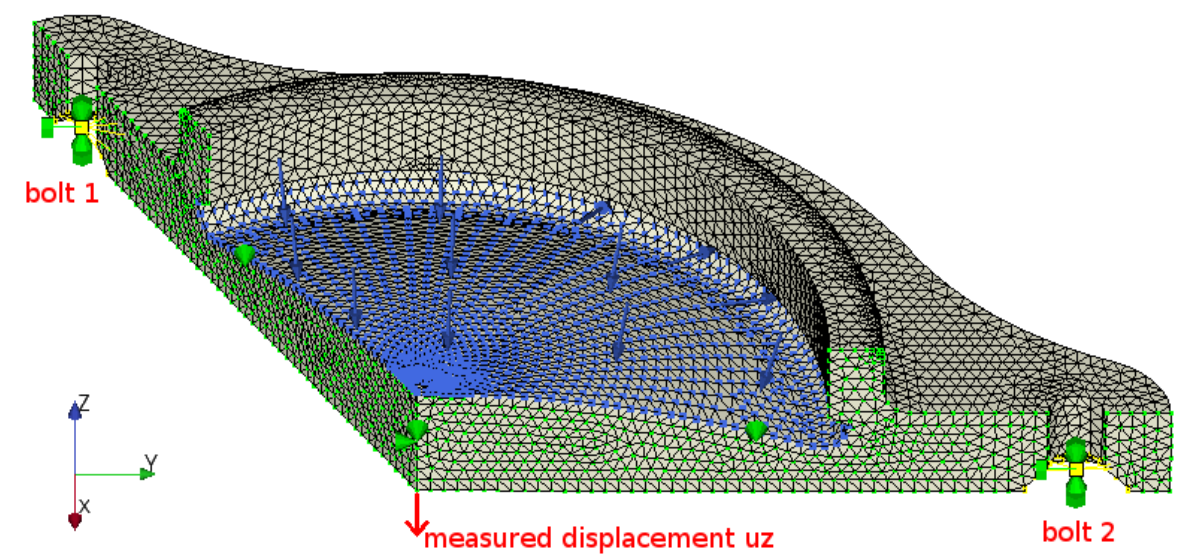

Figure 4: Model with tetra mesh with size $0.5 \mathrm{~mm}$, pressure and symmetry BCs.

\begin{tabular}{|c|c|c|c|}
\hline Variant & Element type & Mesh size $[\mathrm{mm}]$ & Optimization settings \\
\hline A1 & C3D4 (4-node tetra) & 0.5 & Removing (+3 \%, -6 \%) * \\
\hline A2 & C3D4 (4-node tetra) & 0.5 & Growing (+2 \%, -1 \%) \\
\hline A3 & C3D4 (4-node tetra) & 0.25 & Growing (+2 \%, -1 \%) \\
\hline B1 & C3D8I (improved 8-node hexa) & 0.15 & Growing (+2 \%, -1 \%) \\
\hline B2 & C3D8I (improved 8-node hexa) & 0.15 & Growing (+1 \%, -0.5 \%) \\
\hline C1 & C3D8I (improved 8-node hexa) & 0.15 & Growing 1 layer/iteration \\
\hline C2 & C3D8I (improved 8-node hexa) & 0.15 & Growing 1 layer/iteration \\
\hline
\end{tabular}

Table 1: Model variants.

*) In the model A1 steel was being removed from the whole steel model (substituting to copper) by prescribed percentage of the actual steel content. In the other variants steel was growing from the full copper model by the given percentage of the full model volume.

Models B with hexa elements were meshed easily by "body fitting" in the Salome software, so that boundaries of the mesh are as stairs instead of smooth surfaces corresponding with geometry. Hexa elements enabled to use finer mesh without big increase in optimization time.

4-node tetra elements are according to the solver manual considered as too stiff, in general it is recommended to use second order tetra elements. Linear elements were chosen to obtain results in shorter time expecting that model is rather for initial design and stiffness would be compared in a relation to material changes.

\subsubsection{Layer-wise optimization}

Layer-wise optimization aimed again to minimize compliance with specific number of steel layers in each iteration. Variables were set as material in each layer. The $\mathrm{C} 1$ variant used whole base plate as a design domain divided to 43 layers of thickness $0.15 \mathrm{~mm}$ and went from $0 \%$ steel to $50 \%$ of the part volume. Since steel layers in the bottom would decrease heat flow due to poor steel conductivity (16 $\mathrm{W} / \mathrm{mK}$ compared to copper $210-230 \mathrm{~W} / \mathrm{mK}$ ), the more realistic $\mathrm{C} 2$ variant used a design domain limited to the layers above bolt holes in order to unblock thermal path from the bottom side to the conduction cover (Figure 1).

\subsection{Single-material concept}

Figure 5 shows one sixth model. The non-design space consists of quadratic CTETRA elements of size $1 \mathrm{~mm}$. High of the ring was increased by $2.3 \mathrm{~mm}$ as a maximum allowed by the existing heat switch design. The design domain consisted of CHEXA elements of size $0.2 \mathrm{~mm}$. The design domain was in the 
space which is filled with paraffin. Step around the design domain center was made to avoid building large ceiling in the result shape. The ceiling would during manufacturing need supports difficult to remove. Main difference from previous optimizations is that in this case topology optimization was used to create a structure which will ensure $0.5 \mathrm{~mm}$ thin floor to remain within flatness tolerance.
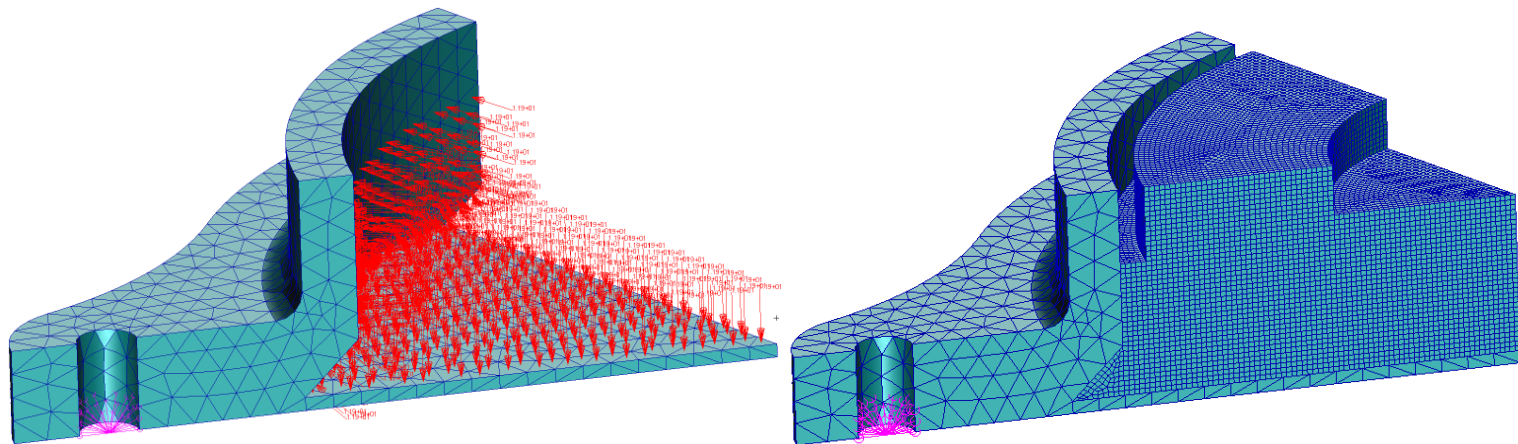

Figure 5: Left: the non-design space with a pressure load, right: whole model with fine mesh in the design space.

Topology optimization was defined to minimize mass subjected to constraints for the nodes under the thin plate (Figure 5 left)

$$
-0.05 m m<u_{z i}-u_{z c}<0.05 \mathrm{~mm}
$$

where $\mathrm{u}_{\mathrm{zi}}$ is the vertical displacement of the $\mathrm{i}$-th node, $\mathrm{u}_{\mathrm{zc}}$ is the vertical displacement of the center node. From manufacturing constraints minimum member size was prescribed to $0.3 \mathrm{~mm}$ and the penalization coefficient was changed from default 3 to 5 to get more compact results.

There are also another design options which are not included in this study but we will mention them in this paragraph. From a manufacturing point of view, it would be favorable to use a lattice structure inside the design domain. That would remove potential difficulties with the manufacturing supports removal from such a small space as is inside the optimized single-material structure. Such optimization example can be found in Tang et al. [17] and a review of additively manufactured lattice structures made Tamburrino et al. [18]. Another approach which could increase the stiffness and could keep a similar weight would be a sandwich concept made by increasing the height of the base plate using a lightweight structure inside the plate separated from the paraffin space.

\section{RESULTS}

\subsection{Multi-material concepts}

\subsubsection{Intuitive approach}

The results from individual simulations are shown in Figure 6, where is the deformation of the base plate bottom in a section through one screw hole as marked in Figure 2; point $[0,0]$ corresponds to the center of the base plate bottom. Decrease of the deflection is not big - model 04 contains $12.6 \%$ of steel instead of copper and center point deflection is $85 \%$ of the non-reinforced model. 


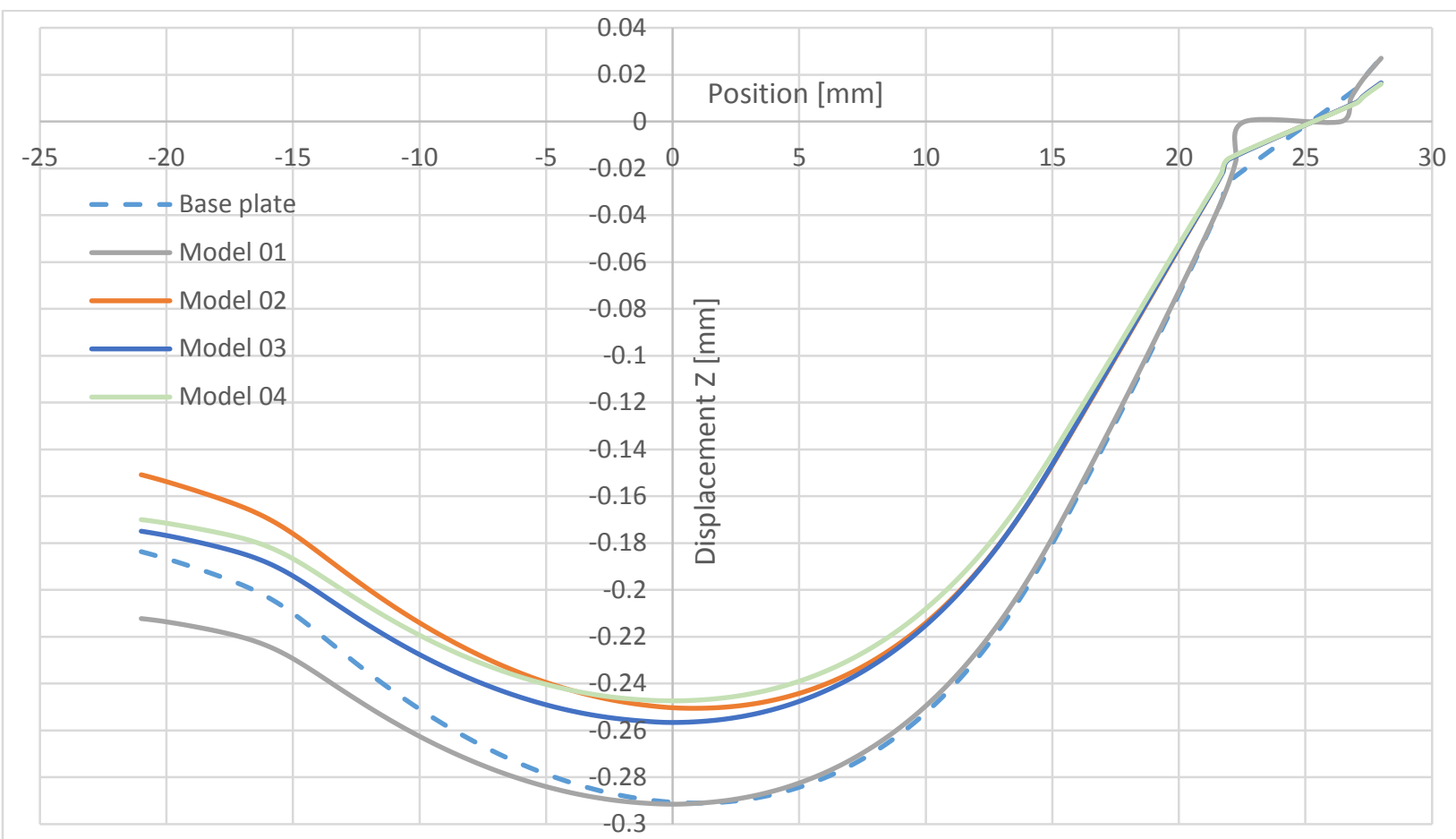

Figure 6: Deflection of the Base plate bottom in the plane marked in Figure 2.

\subsubsection{Topology optimization}

Gained decrease of the displacement is in Table 2 and the graph in Figure 7. With the higher steel content the effect of reinforcing slows down. All models give very similar results. Reinforcements are primarily around bolts and creates top "flange" of a "beam" between bolts. Secondary reinforcement is in the middle of a "plate" surface creating a sandwich like structure.

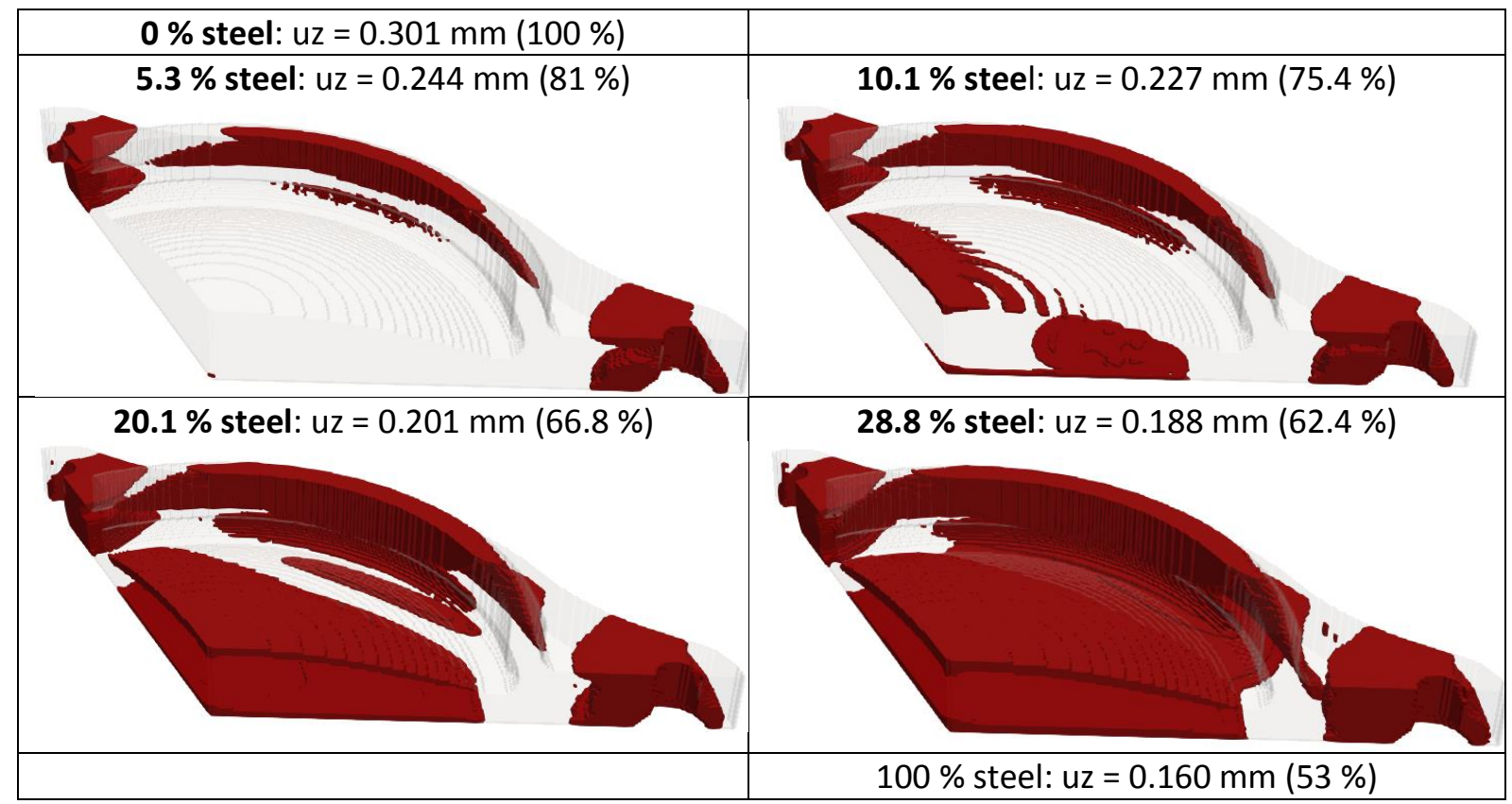

Table 2: Different steel volume contents with the hexa mesh model B2, red - steel, copper is transparent.

From the graph in Figure 7 it is visible that there is not significant difference between results $A 1$ and $A 2$, and between $B 1$ and $B 2$, so that optimization settings are equivalent (removing or growing of the model A1 vs. A2, and chosen speeds of changes B1 vs. B2.). Finer tetra mesh gives different 
displacements in comparison to rough tetra mesh. By comparison of A3 and B2 results in the table it is worth of noticing that the tetra mesh is stiffer by $5.6 \%$ but relative changes are similar (within $1.3 \%$ ). Optimization leads also to improvement of global strenght thanks to higher strenght of reinforcing steel in compare to copper and fact that reinforcement is placed in the areas where original copper material was more stressed.

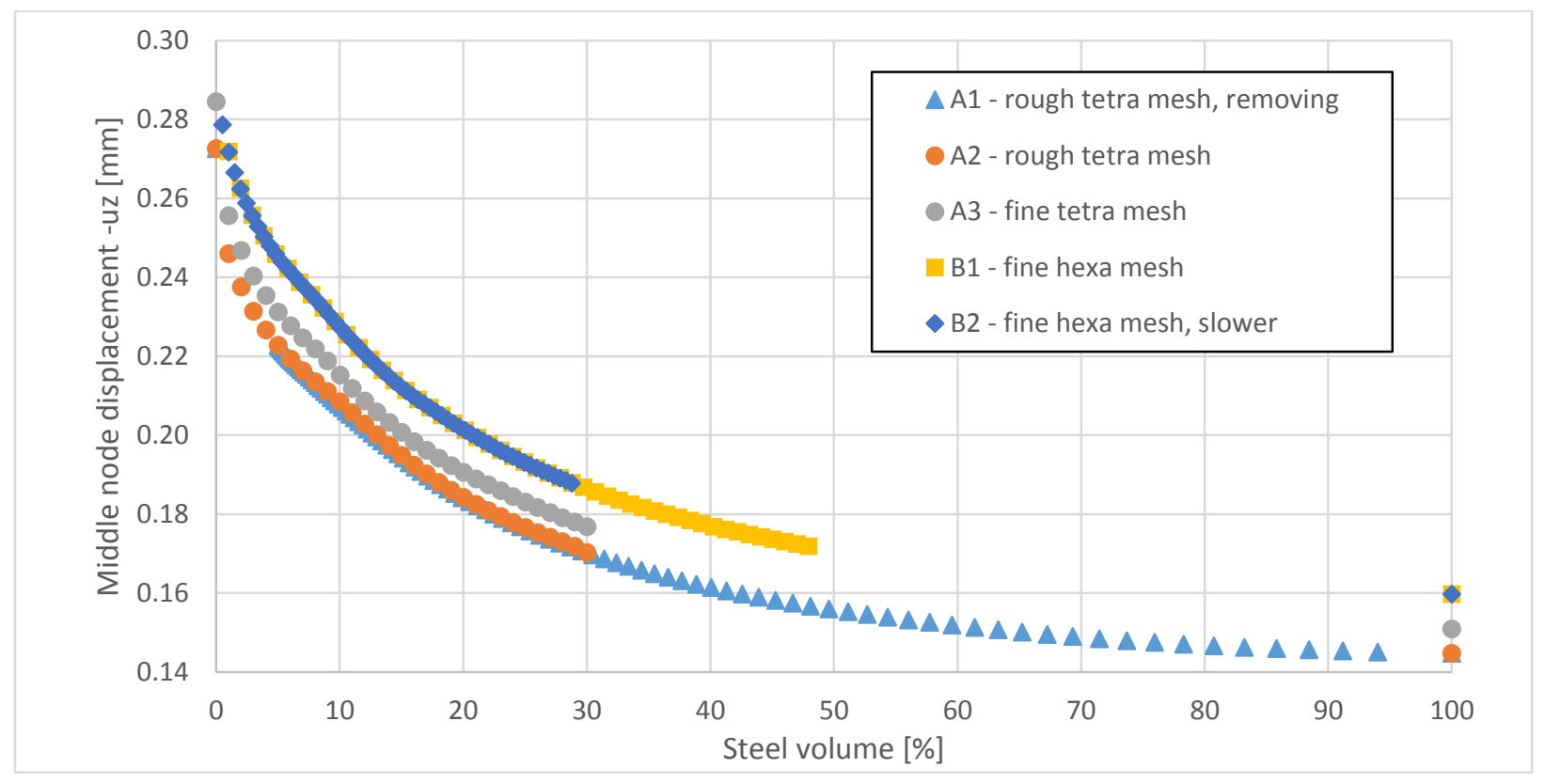

Figure 7: Centre node displacement vs. steel volume.

\subsubsection{Layer-wise optimization}

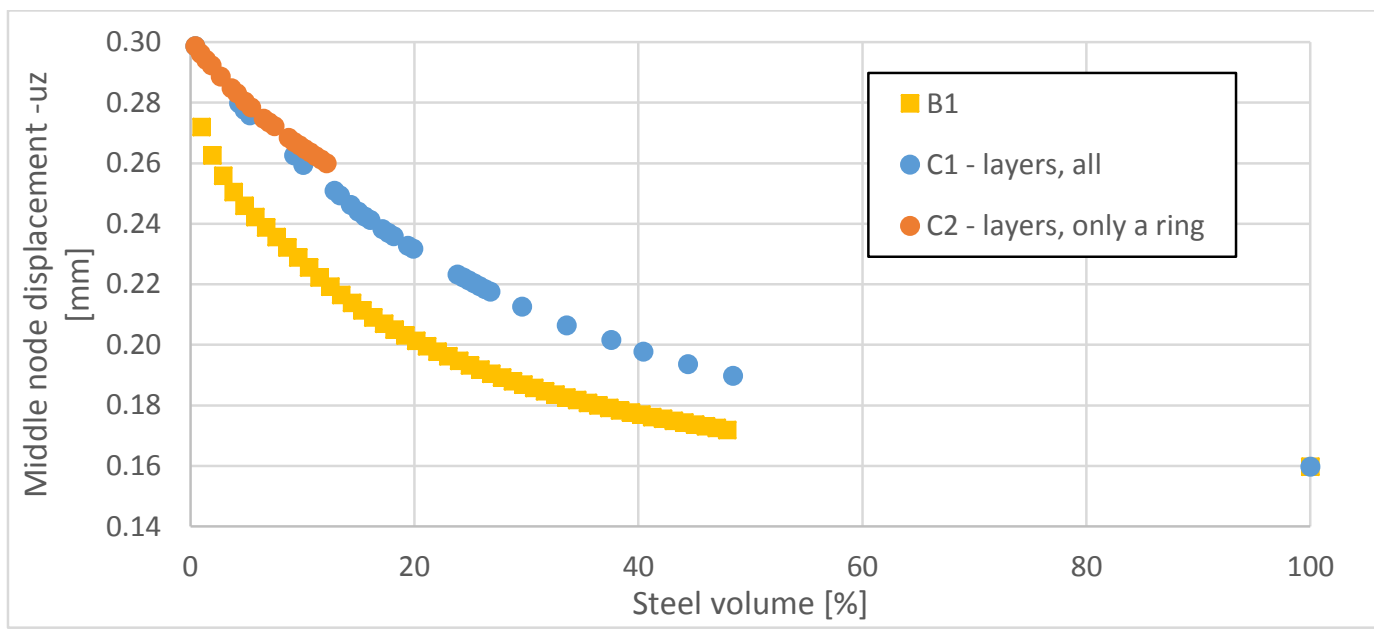

Figure 8: Centre node displacement vs. steel volume, B1 model is for reference.

Efficiency of stiffness increase was smaller in case of layer-wise optimization as shown in Figure 8, e.g. for the $5 \%$ steel B1 model deformed by $81.3 \%$ and the C1 model deformed approximately by $92 \%$. The variant $\mathrm{C} 1$ added steel to the ring area and to the bottom. The stiffness difference to $\mathrm{C} 2$ variant is not so dramatic as can be read from Table 3 . 


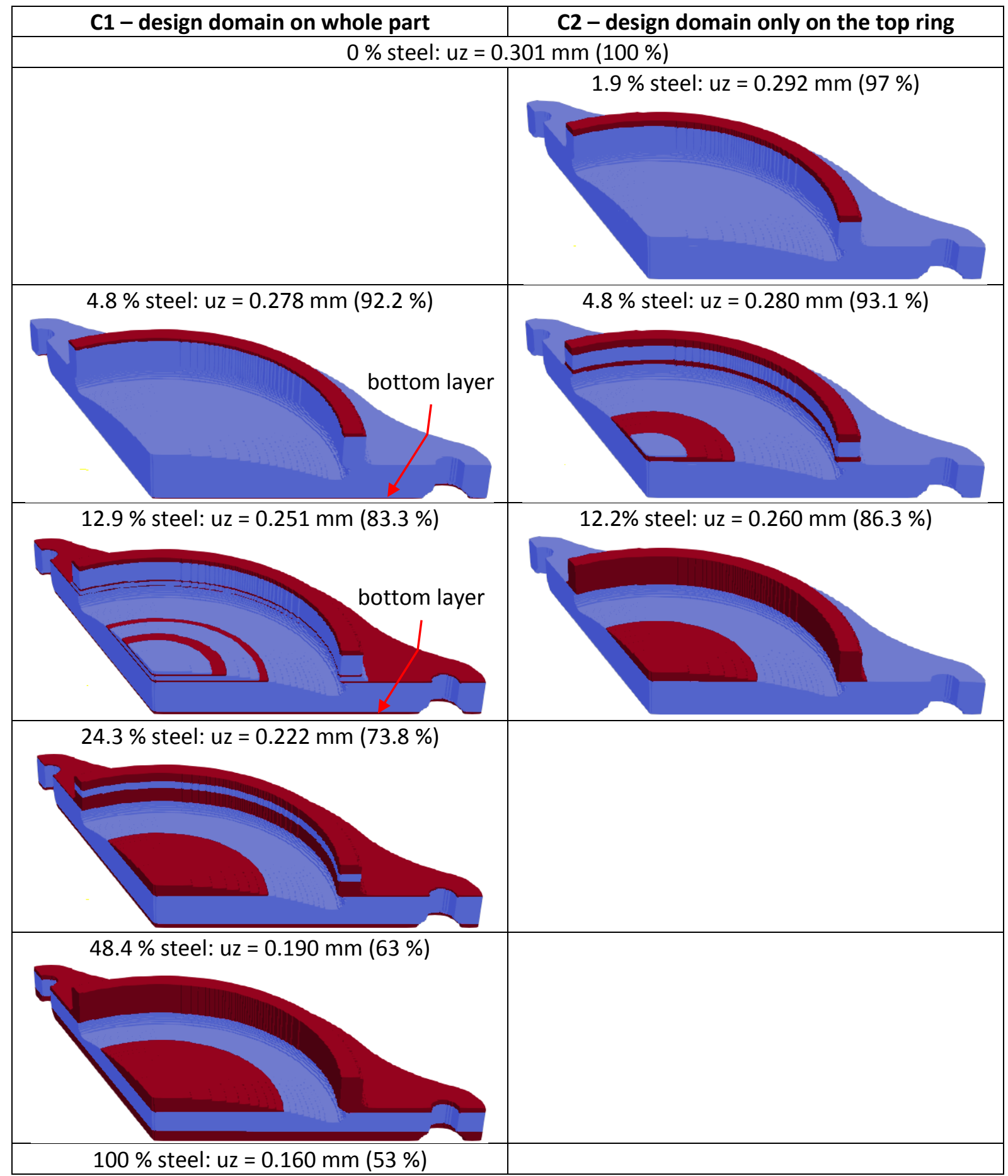

Table 3: Visual comparison of models C1 and C2, red - steel, blue - copper.

\subsection{Single-material concept}

The resulting shape after smoothing is in the Figure 8. A user must choose the threshold to take elements as solid or void without intermediate pseudo-densities, because original result is not truly discrete, so there is a little disproportion between the result in optimization and after recalculation with discrete (real) material. Discrete model was slightly stiffer then the result from "shadow" density optimization. Final flatness of the bottom is showed in Figure 9. Obtained topology is, contrary to higher ring, lighter then original single material model ( $43.1 \mathrm{~g}$ vs. $51.9 \mathrm{~g}$ ), but it is overstressed with concentrations up to $1600 \mathrm{MPa}$. Stress constraints were not applicable in Nastran due to stress 
concentration around fixed nodes. So the result would require to add material, e.g. by shape optimization.
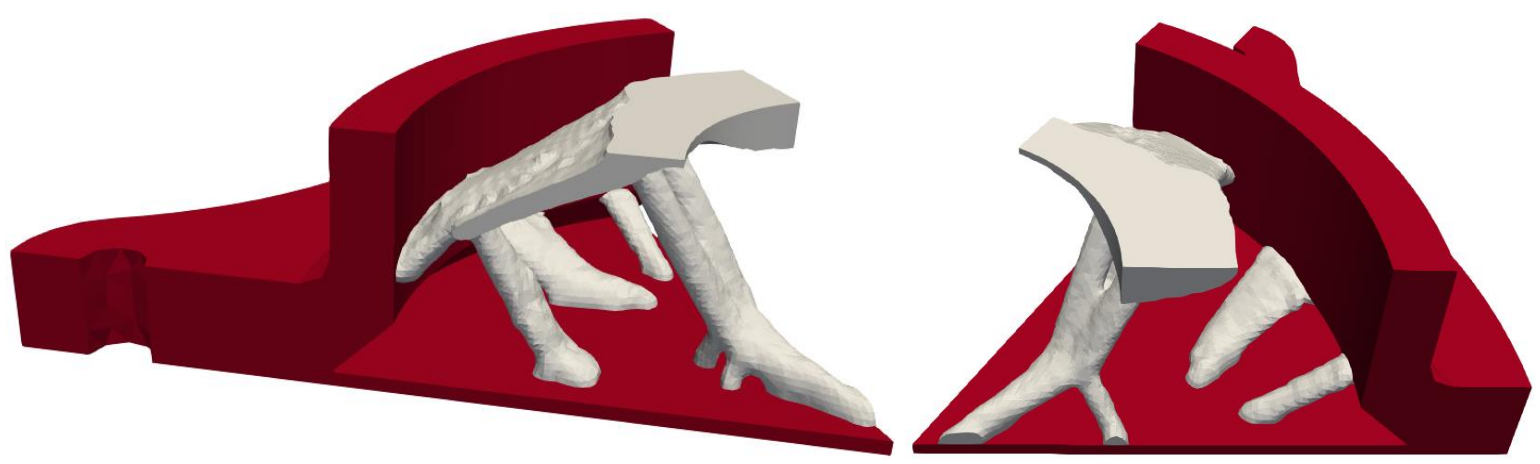

Figure 8: Views on the smoothed resulting shape.
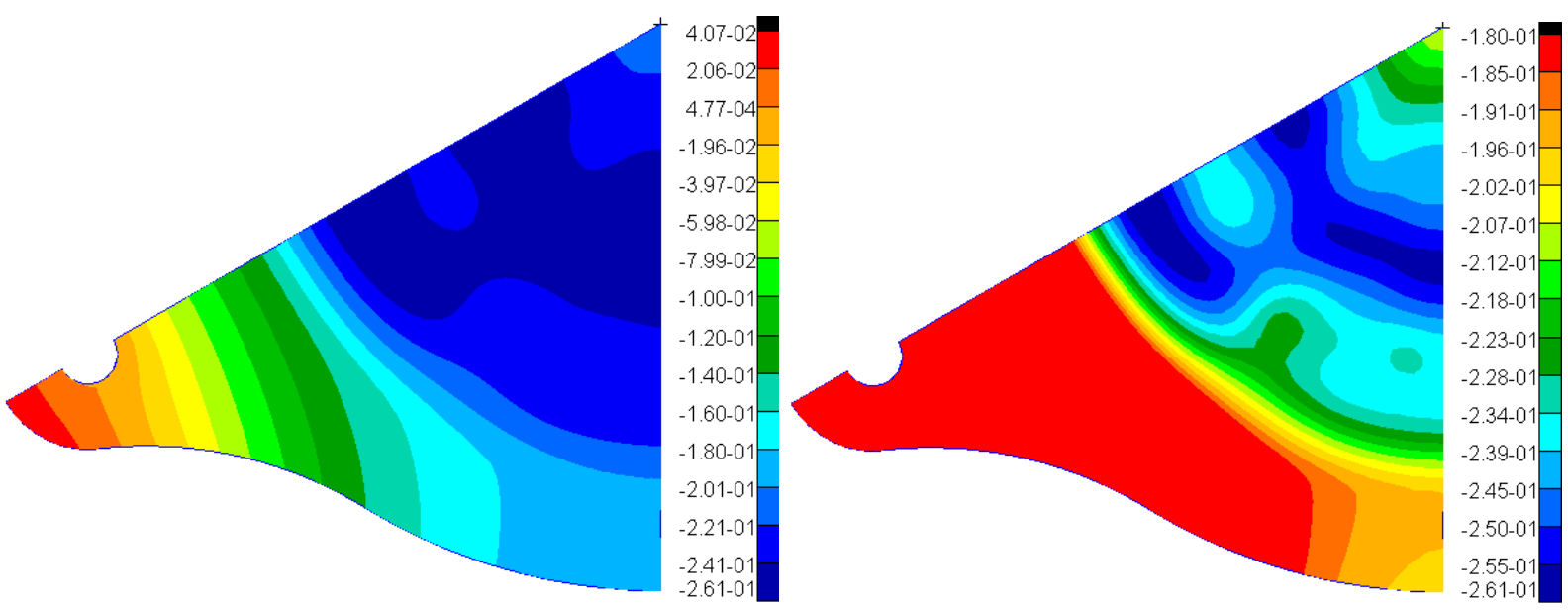

Figure 9: Vertical displacement [mm] of the bottom, on right side the fringe is limited to highlight center area where nodes had design constrains.

\subsection{Comparison}

Deflections in the bottom cross section (as marked in Figure 2) are compared in Figure 10 for the representative models. The lowest deflection (by $22 \%$ lower than the original) has the model B2 from topology optimization with multi-materials where $10.1 \%$ of steel was chosen since it does not yet cover large surfaces with steel preventing heat transfer (as shown in Table 2). Second lowest deflection (by $15 \%$ lower than the original) has the Model 04 from the multi-material intuitive design (Fig. 3). Those designs would require multi-material AM. If we will limit AM to print layers with consistent material, then model C2 (with $12.2 \%$ of steel in Table 3) from the layer-wise optimization has deflection $10.7 \%$ lower than the original.

Single-material concept with modified design domain geometry is manufacturable with classical SLM method. With the prescribed flatness of the bottom it has relatively wavy curve due to the big flatness tolerance but after touching counterpart a contact area might be much larger thanks to deformation of weak floor of this model. This model is also lighter but local stress concentrations would probably require to add material. Difficulties can arise during filling inner space with paraffin because of the complicated structure inside.

Our work covered only stiffness study but in continuation thermo-mechanical study should compare base plate designs in the whole heat switch model to consider total thermal transfer through the whole assembly. 


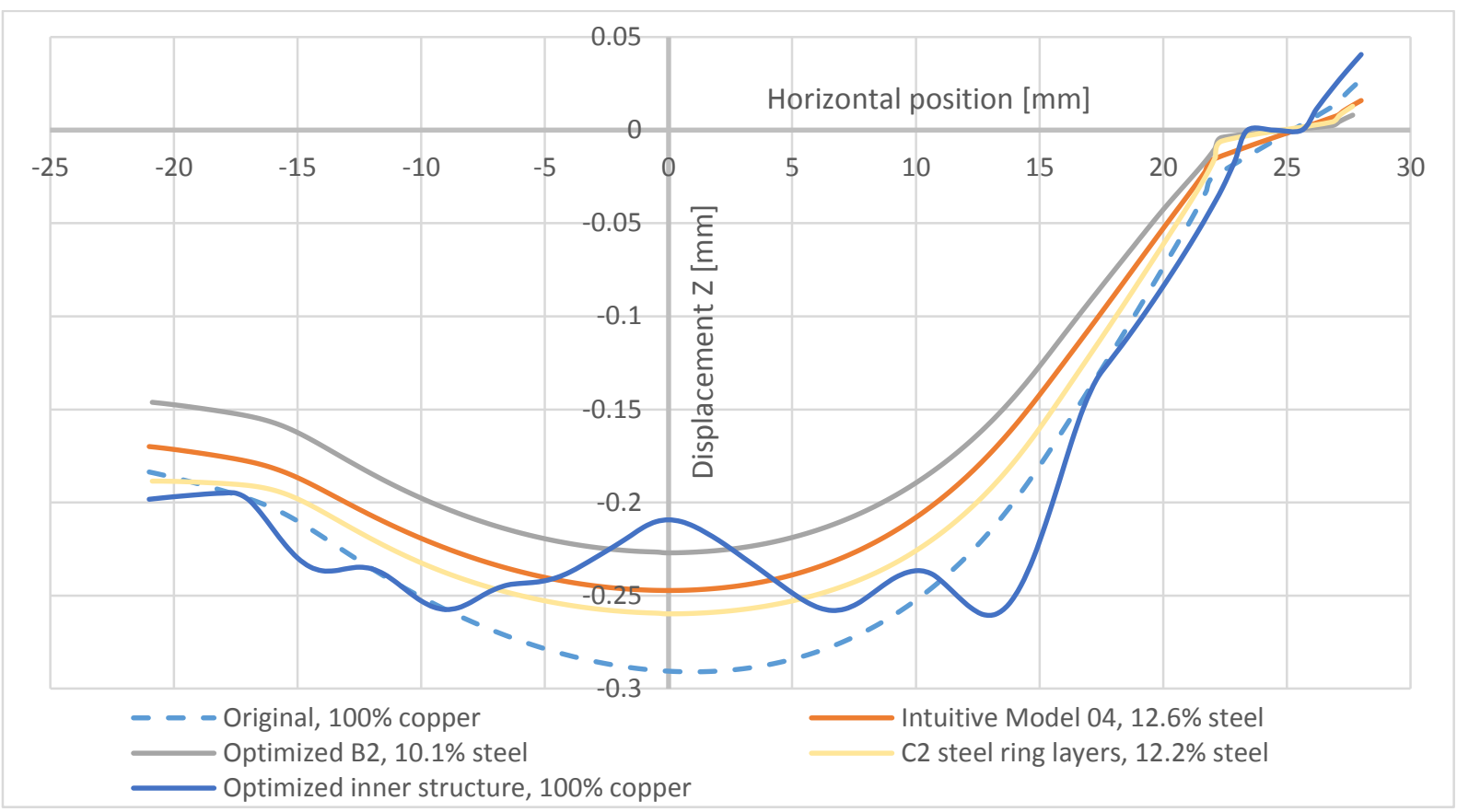

Figure 10: Deflection comparison of the explored concepts in cross section as marked in Figure 2.

\section{CONCLUSION}

Several variants of the base plate were investigated in order to increase contact area under the miniaturized heat switch. Multi-material designs with original geometry demonstrated the deflection decrease dependent on the prescribed volume of reinforcing steel. Deflection curves were compared for the optimized design requiring AM of steel and copper in one layer, design limited to one material in the layer which is easier to manufacture, and design from single-material optimization with flatness constraints. The latter solution has the lower weight, but stress concentrations need attention and filling of paraffin should be checked. Detailed thermal analysis was left for further analysis.

\section{ACKNOWLEDGEMENTS}

The work was supported by the European Space Agency project "Additive Design for Aerospace Applications Capabilities", Contract No. 4000123317/18/NL/GLC/hh.

\section{REFERENCES}

[1] Mašek, J., Brožek, P., Horák, M. (2017). Thermo-Vacuum Test Chamber Development for Heat Switch Testing in Simulated Martian Conditions. 23 $3^{\text {rd }}$ International Conference Engineering Mechanics, Svratka, Czech Republic, 15-18 May 2017. 622-625.

[2] Mašek, J., Popela, R. (2018). Heat Switch Design Overview \& Advanced MHS Definition. Technical note. Institute of Aerospace Engineering, Faculty of Mechanical Engineering, Brno University of Technology.

[3] F2792 - 12a, Standard Terminology for Additive Manufacturing Technologies (2012), ASTM International, West Conshohocken, PA.

[4] Scott, J., Gupta, N., Weber, C., Newsome, S., Wohlers, T., Caffrey, T. (2012). Additive Manufacturing: Status and Opportunities. IDA Science and Technology Policy Institute. 
[5] Ngo, T. D., Kashani, A., Imbalzano, G., Nguyen K. T.Q., Hui, D. (2018). Additive Manufacturing (3D printing): A Review of Materials, Methods, Applications and Challenges. Composites Part B: Engineerign, 143, 172-196.

[6] Frazier, W. E. (2014). Metal Additive Manufacturing: A Review. Journal of Materials Engineering and Performance, 23(6), 1917-1928.

[7] Mahamood, R. M., Akinlabi, E. T. (2017). Functionally Graded Materials. Springer International publishing AG. ISSN 2364-3307, ISBN 978-3-319-53756-6.

[8] Obielodan, J. O., Ceylan, A., Murr, L. E., Stucker, B. E. (2010). Multi-material Bonding in Ultrasonic Consolidation. Rapid Prototyping Journal, 16(3), 180-188.

[9] Chivel, Y. (2016). New Approach to Multi-material Processing in Selective Laser Melting. 9th International Conference on Photonic Technologies - LANE 2016. Physics Procedia, 83, 891-8980.

[10] Wei, C., Li, L., Zhang, X., Chueh, Y-H. (2018). 3D printing of Multiple Metallic Materials via Modified Selective Laser Melting. CIRP Annals, 67(1), 245-248.

[11] Bensøe, M. P. (1989). Optimal shape design as a material distribution problem. Structural and Multidisciplinary Optimization, 1(4), 193-202.

[12] MSC Nastran 2018: Design Sensitivity and Optimization, User's Guide. MSC Software

[13] Bensøe, M.P., Sigmund, O. (2004). Topology optimization: theory, methods and applications. Second edition. Berlin: Springer, 2004, 370 p. ISBN 3-540-42992-1.

[14] Sigmund, O., Torquato, S. Design of materials with extreme thermal expansion using a threephase topology optimization method. Journal of the Mechanics and Physics of Solids, 45(6), 10371067.

[15] Xia, L., Xia, Q., Huang, X., Xie, Y.M. (2018). Bi-directional Evolutionary Structural Optimization on Advanced Structures and Materials: A Comprehensive Review. Archives of Computational Methods in Engineering, 25(2), 437-478.

[16] Löffelmann, F. (2017). Failure Index Based Topology Optimization for Multiple Properties. $23^{\text {rd }}$ International Conference Engineering Mechanics, Svratka, Czech Republic, 15-18 May 2017. 590593.

[17] Tang, Y., Kurtz, A., Zhao, Y. F. (2015). Bidirectional Evolutionary Structural Optimization (BESO) based design method for lattice structure to be fabricated by additive manufacturing. ComputerAided Design, 69, 91-101.

[18] Tamburrino, F., Graziosi, S., Bordegoni, M. (2018). The Design Process of Additively Manufactured Mesoscale Lattice Structures: A Review. Journal of Computing and Information Science in Engineering, 18.

\section{COPYRIGHT STATEMENT}

The authors confirm that they, and/or their company or organization, hold copyright on all of the original material included in this paper. The authors also confirm that they have obtained permission, from the copyright holder of any third party material included in this paper, to publish it as part of their paper. The authors confirm that they give permission, or have obtained permission from the copyright holder of this paper, for the publication and distribution of this paper as part of the READ 2018 proceedings. 
This is an open access article distributed under the Creative Commons Attribution License which permits unrestricted use, distribution, and reproduction in any medium, provided the original work is properly cited. (CC BY 4.0). 